\title{
NECROSE DA MUCOSA ESOFÁGICA COMO COMPLICAÇÃO DA CARDIOMIOTOMIA À HELLER PARA TRATAMENTO DE MEGAESÔFAGO CHAGÁSICO
}

\author{
NECROSIS OF ESOPHAGEAL MUCOSA AS A HELLER CARDIOMYOTOMY \\ COMPLICATION IN THE TREATMENT OF ACHALASIA IN CHAGAS' DISEASE
}

\author{
Nádia Barbosa Aires ${ }^{1}$ \\ Carlos Augusto Teixeira Cruz, TCBC-DF ${ }^{2}$ \\ André Luiz Vianna, TCBC-DF ${ }^{2}$
}

\section{INTRODUÇÃO}

A cardiomiotomia à Heller por videolaparoscopia é opção para tratamento do megaesôfago e apresenta bons resultados, com melhora importante da sintomatologia mesmo a longo prazo ${ }^{1,2}$. Entretanto, observa-se que o procedimento esteve associado, inicialmente, a considerável morbidade, possivelmente devido à curva de aprendizado $^{3}$. O objetivo do presente estudo é relatar complicação pós-operatória da cardiomiotomia à Heller laparoscópica.

\section{RELATO DO CASO}

Paciente masculino, 41 anos, com disfagia progressiva há 15 anos, e diagnóstico pré-operatório de megaesôfago não avançado. Foi submetido a cardiomiotomia à Heller com fundoplicatura parcial por via videolaparoscópica, sem intercorrência perioperatória, e recebeu alta no 2 dia após a operação. No $10^{\circ}$ dia pós-operatório apresentou melena e foi submetido a endoscopia digestiva alta, que evidenciou extensa úlcera na transição esofagogástrica, com cerca de $6 \mathrm{~cm}$ de extensão. Não havia sangramento ativo e optou-se pelo tratamento conservador. No $13^{\circ}$ dia pós-operatório apresentou volumosa hematêmese, que não respondeu ao tratamento clínico. Foi submetido a laparotomia exploradora observando-se necrose da mucosa esofágica na extensão da miotomia. Identificada artéria na parede gástrica, próximo ao vértice da miotomia, que apresentava sangramento ativo. Procedeu-se à hemostasia e à cardioplastia à Thal. Houve boa evolução pós-operatória, com alta no $10^{\circ}$ dia após a reoperação.

\section{DISCUSSÃO}

A cardiomiotomia à Heller por laparoscopia é procedimento recente e com bons resultados ${ }^{2}$. As complicações são raras quando em mãos experientes e as mais observadas são a perfuração da mucosa esofágica e pneumonia ${ }^{4,5}$. O sangramento gastroesofágico também é complicação já relatada, ocorrida duas semanas após a operação em úlcera imediatamente acima da junção gastroesofágica, porém sem a necessidade de tratamento cirúrgico ${ }^{4}$.

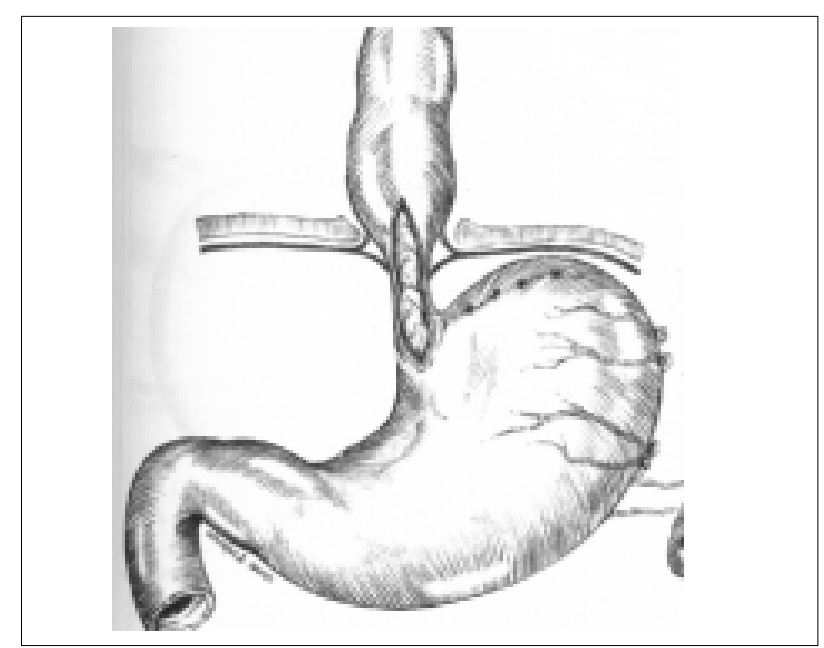

Figura 1 - Cardiomiotomia envolvendo esôfago terminal e cárdia, direcionada à grande curvatura, se afastando da pequena curvatura para evitar os ramos esofagianos da artéria gástrica esquerda.

1. Acadêmica da Faculdade de Medicina da UnB

2. Professor Adjunto da Área de Clínica Cirúrgica da Faculdade de Medicina da UnB

Recebido em 13/11/2001

Aceito para publicação em 18/06/2002

Trabalho realizado no Serviço de Cirurgia Geral do Hospital Universitátio da Universidade de Brasília (UnB) 
No presente estudo, o paciente apresentou quadro semelhante de hemorragia digestiva alta após 10 dias da operação. Entretanto, a hemorragia não respondeu ao tratamento clínico e o paciente foi reoperado, agora por laparotomia. $\mathrm{O}$ achado cirúrgico à macroscopia foi de necrose da mucosa na área da miotomia, seguido de lesão de artéria da parede gástrica, possilvemte por ação das enzimas gástricas.

Outra possível explicação para a complicação observada seria a realização da miotomia na porção gástrica muito próxima à pequena curvatura, provavelmente por exposição insuficiente da junção esofagogástrica durante a videolaparoscopia, atingindo ramos esofágicos da artéria gástrica esquerda (Figura 1). Isto poderia explicar, em parte, a necrose da mucosa esofagogástrica, e a exposição dos vasos da parede gástrica às enzimas do estômago.

A fundoplicatura íntegra diminuiu a morbidade da complicação pós-operatória no presente caso, e a reoperação pela técnica de Thal proporcionou bons resultados, mesmo sendo realizada em condições adversas. Não foram encontrados na literatura casos de complicações semelhantes.

\begin{abstract}
This article presents a complication of the laparoscopic technique for Heller cardiomyotomy and anterior fundoplication. This procedure is safe and provides excellent relief of disphagia in esophageal achalasia. Nevertheless, there are rare but dangerous complications, such as late active digestive bleeding, presented in this paper which was resistant to conservative treatment and led to hypovolemic shock. Urgent laparotomy performed to identify and control bleeding, revealed necrosis of esophageal mucosa with a bleeding gastric vessel. Inadequate exposure of the gastroesophageal junction and an incision very close to the lesser curvature might have damaged the esophageal branches of the left gastric artery, leading to ischemic necrosis of the mucosa and exposure of the gastric wall and its vessels.
\end{abstract}

Key Words: Achalasia; Heller cardiomyotomy; Laparoscopic cardiomyotomy; Myotomy complications.

\title{
REFERÊNCIAS
}

1. Yamamura MS et al. Laparoscopic Heller myotomy and anterior fundoplication for achalasia results in a high degree of patient satisfaction. Arch Surg 135:902-906, 2000.

2. Spiess AE, Kahrilas PJ. Treating achalasia: from whalebone to laparoscope. JAMA 280:638-642, 1998.

3. Soot $\mathrm{J}$ et al. Transition from open to laparoscopic fundoplication: the learning curve. Arch Surg 134:278-281, 1999.

4. Hunter JG et al. Laparoscopic Heller myotomy and fundoplication for achalasia. Ann Surg 225:655-665, 1997.
5. Herbella FAM et al. Resultados tardios da operação de Heller associada à fundoplicatura no tratamento do megaesôfago: análise de 83 casos. Rev Ass Med Brasil 45:317-322, 1999.

Endereço para correspondência:

Nádia Barbosa Aires

QSA 13 casa 15

72015-130 - Taguatinga-DF

e-mail: virgolino@terra.com.br 